Aus dem Protozoen-Laboratorium des Kaiserlichen Gesundheitsamtes. (Vorsteher: Reg.-Rat Dr. Schaudinn.)

\title{
Untersuchungen über das Wesen des Vaccineerregers.
}

Vorläufige Mitteilung von Dr. S. Prowazek (Rovigno), Hilfsarbeiter am Kaiserlichen Gesundheitsamte.

Seit der im Jahre 1901 erschienenen kritischen Untersuchung von v. Wasielewski über die Natur des Vaccineerregers wurde abermals eine weitere Reihe von . Irbeiten (Dombrowski, Ishigami, Bosc, Thomson, Browne, Councilman, Magrath, Brinckerhoff, Calkins, Foa, Siegel u. a.) publiziert, durch die teilweise, der von v. Wa. sielewski gewonnene Standpunkt: „Lie von Guarnieri entdeckten Vaccinekörperchen sind sehr wahıscheinlich die Vaccineerreger selbst", verlassen wurde, während andererseits, angeregt durch die inzwischen vertiefte Protozoenforschung, andere Fragestellungen in den Vordergrund des wissenschaftlichen Interesses gerückt wurden. Alle diese Momente machten eine Nachuntersuchung der Vaccinefrage notwendig.

I. In erster Linie wurde die zum Teil von der $\mathbf{k}$. k. Impfstoffgewinnungsanstalt in Wien, zum Teil von M. Lang in Triest bezogene Lymphe sorgfältig mikroskopisch untersucht. Zur Untersuchung kamen entweder frische Deckglaspräparate oder Ausstriche (Fixierung: $2 / 3$ konzentriertes Sublimat und $1 / 3$ $90 \%$ igen Alkohol [heiB], Färbung: verdünntes Grenachers Hämatoxylin). Neben allerhand undefinierbarem Zelldetritus, Plasmafasern, Tröpfchen und Kügelchen waren in allen untersuchten Lymphsorten in den kleinsten Epithelfragmenten mit Hämatoxylin färbbare Gebilde nachweisbar, die ich vorläufig mit dem völlig indifferenten Namen "Lymphkörperchen“ bezeichnen will.

Sie sind rundlich oder meist oval, sehr deutlich konturiert und führen in ihrem hellen, fast strukturlosen Inhalt ein bis zwei $(1-11 / 2 \mu$ große) färbbare, dunkle Einschlüsse, die selten punktförmig, meist stäbchenartig oder keilförmig sind. Die längeren Formen sind zuweilen in der Mitte leicht eingeschnürt oder geknickt (Winkelstellung). Bei einer 2250fachen Vergrößerung kann man gerade noch einzelne Alveolenzüge 
wahrnehmen, die zu der peripheren Kontur verlaufen. Sie bilden einen charakteristischen Bestandteil der Lymphe. Wurden aus der mit physiologischer Kochsalzlösung verdünnten Lymphe mit feinsten Glasfäden auf die Spitze eines geeigneten Glassplitters (Deckglas) einzelne Lymphkörperchen isoliert, das Deckglasfragment mittels Wachs auf einen ausgehöhlten Objektträger montiert und mit geeigneten Vergrößerungen die Zahl der fraglichen Körperchen festgestellt (z. B. 14 oder 20 etc.), so konnte man hernach mit der Deckglasspitze mit Erfolg in eine Corneatasche des Kaninchenauges eine Impfung vornehmen. Gegen dieses Experiment kann man allerdings den Einwand erheben, daß mit den Lymphkörpern der ultravisible Erreger mit verimpft wurde.

II. Mit der Lymphe wurden auch Verdünnungsexperimente vorgenommen; sie ist in einer Verdünnung von $1: 1000 \mathrm{ge}$ lade noch wirksam. Daß der Erreger bei dieser Verdünnung korpuskulär in einem über einen größeren Raum verteilten $\mathrm{Zu}$ stand vorkommt, dafür spricht der Umstand, daß nach der Verimpfung in der kreuz- und quergestrichelten Cornea anfangs nur lokal einzelne isolierte Trübungen entstehen können. Auch kann nach einer Filtration des Lymphmaterials durch ein mehr als vierfach gefaltetes Filterpapier mittels Nadeln aus ein- und demselben Filtrat bald mit, bald ohne Erfolg die Cornea geimpft werden.

III. Um den Nachweis zu erbringen, daß in der verdünnten lyynphe deutlich bewegliche (nicht Brownsche Bewegung) Entwicklungsstadien vorkommen. eine Annahme, die die letzten Beobachtungen von Siegel zu stützen schienen, wurden folgende Experimente angestellt:

a) Die Lymphe wurde sehr vorsichtig mit Kapillarröhrchen auf den Boden von zugespitzten Zentrifugiergläschen, die mit physiologischer Kochsalzlösung gefüllt waren, gebracht, wolauf die daruiber lagernde Flüssigkeit in einem Zeitraum von acht Stunden bis mehreren Tagen, stufenweise verimpit wurde.

b) Die Lymphe wurde mit angeritzten Kapillaren auf den Boden der Glasgefäße gebracht, worauf durch einen leichten Fruck der untere Teil der Kapillare abgebrochen wurde. Das weitere Verfahren war wie im ersten Fall.

c) Schlieblich wurde die Lymphe in kleinen, mit Filterpapier verschlossenen Glastuben auf den Boden der Gläser versenkt, worauf mit del darüberlagernden Flüssigkeit in entsprechenden Zeiträumen Impfversuche vorgenommen wurden

Alle diese Versuche führten zu dem Resultat, daß in der ciberen Flüssigkeitszone keinerlej intensiver bewegliche Formen vorkommen. Für die Trennung des Virus vom Medium künnte man sich auch des Sedimentierungsverfahrens oder der Zentrifugiermethode (Pfeiffer, Bonhoff) bedienen; das erstere Verfahren ist aber für die Lymphe eine langwierige, nie exakt durchführbare Prozedur; der letzteren Methode konnte ich mich nicht bedienen, da mir keine so starke Zentrifuge zur Verfügung stand.

IV. Nach der Impfung findet man in der Cornea des Kaninchens in sich stetig steigernder Menge, von der dritten Stunde ab angefangen, die bekannten, vielfach beschriebenen Guarnierischen Körper, daneben aber auch andere, allerdings sehr schwer nachweisbare Gebilde, die ich Initialkörper nennen will. Einzelne wurden bereits von Bosc im Zentralblatt für Bakteriologie Bd. 37, H. 2, Taf. 1, Fig. 23, abgebildet. Sie sind zumeist oval, $1-11 / 2 \mu$ groß und schließen in einem hellen Hof zwei meist verschieden große chromatische Körnchen ein. Man kann sie am besten darstellen, wenn man die höchstens $4-5 \mu$ dicken Schnitte mit Saffranin, Victoriablau, Dahlia oder Gentianaviolett stark färbt, entsprechend differenziert und dann mit einer $10 \%$ igen wässerigen Tanninlösung, hernach mit $1 \%$ iger wässriger Brechweinsteinlösung behandelt. Auch die Giemsa-Mischung, in der die Schnitte iiber Nacht belassen wurden, eignet sich zu ihrer Darstellung. An geeigneten Präparaten sieht man in den Guarnierischen Körpern zentral ähnliche, oft eingeschnürte, hantelförmige Gebilde (vgl. die Photogramme 27, 28, 29-33 in der Arbeit von Siegel: Untersuchungen über die Aetiologie der Pocken und der Maul- und Klauenseuche. Anhang zu den Abhandlungen der Königl. PreuB. Akademie 1905). Sie führen in älteren Stadien der Guarnieri- schen Körper lebhafte Bewegungen aus. Die jüngsten Guarnierischen Körpel liegen dieht am liern. in einigen -allerdings äußerst seltenen - Fällen wurden sie auch im Kerm beobachtet. Manche hängen mittels einer schmalen Brïicke mit der Kernmeinbran zusammen - Bilder. die auf einell Austritt derselben hinweisen dürften. Ihre weiteren Velïllderungenl wurden bereits von Hückel zutreffend beschrieben und abgebildet. Auf den späteren Stadien kann man in ihnen eine Zusammensetzung aus zwei Componenten beobachten: die eine hat eine große Vel'wandtschaft zu dem Chromatin. während die andere mit dem Plastin verwandt sein dürtte.

Das Verhalten der Guarnierischen Körper den gebräıchlichen Chromatin- und Kernstoffreaktionen gegellübel ist folgendes:

Mit Methylgrünessigsäure („Unter den basischen Farbbstoffen könnte man mit eineln Schein von Berechtigung nur das Methylgrün als Kernfarbstoff bezeichnen", A. Fischel') färben sie sich wie die Kerne und widerstehen wie diese lange der Verdauung mit Trypsin; mit $20 \%$ iger Kochsalzlosullg werden sie deformiert; manche schwellen an; meist ist aber il einer Vacuole ein chromatischel Brocken lange Zeit nachweisbal"; mit $40 \%$ iger Pottaschelösung ( $21 / 2$ Tage) werden sie bis auf wenige blasse chromatische Bestandteile gelöst. Lasselbe gilt von der verdünnten Kalilauge. Das von Schwarz zum Studium des Chromatins empfohlene Reagens: 1 Volumen $10 \%$ ige Blutlaugensalzlösung, 2 Volumen Wasser, 1/2 Volumen Eisessig, lieferte keine Resultate. Die Berlinerblau-Methode von List zum Nach weis derNukleolarsubstanzen fiel negativ aus.

V. Die Guarnierischen Körper können nicht selbst die Parasiten sein; denn man kann sie nach dem Vorgang von $\mathrm{Foa}$ mit einer $10 \%$ igen Kochsalzlösung im weitgehenden Maße schädigen und doch mit dem derart einen Tag lang volbehandelten Material eine elfolgreiche Impfung vornehmen. Dasselbe gilt für $20 \%$ ige und konzentrierte liochsalzlösung, sowie Trypsin (nit 0,5 Sodalösung 24 Stunden). das nicht encystierte Protozoen sicher vernichten würde.

VI. Die fraglichen Körper sind, wie bereits aus den Untersuchungen von Wasielewski hervorgeht, für die Vaccine spezifisch und besitzen höchstens mit den Negrischen Körpern der Tollwut eine gewisse Aehnlichkeit: weder nach einfachen Verletzungen noch nach einer kombinierten Nachbehandlung mit Glycerin oder. Trypsin können ähnliche Gebilde in so typischel. Weise und Verteilung elzeugt werden. In dieser Beziehung sagt J ür ge n s mit Recht: „ Selbstverständlich können Täuschungen vorkommen. Zertlümmerte Epithelzellen oder eingewanderte Leukocyten können manchnal als ithnliche Gebilde imponieren." - Es geht daher nicht an, auf die Anwesenheit eines oder einiger nicht näher definierbarel. rundlicher Gebilde hin die Impfung als positiv zu bezeichnen.

Aus den Reaktionen zu schließen (Methylgrünessigsäure, Trypsin, 40\% ige Pottasche, Salz etc.), sind sie mit den allerdings chemisch heutzutage noch weniger als früher scharf definierbaren Kernsubstanzen (man vergleiche die verschiedenen Nebenkerne, Mitochondrienkörper [Goldschmidt] etc.) verwandt, die nach den neueren Forschungen im weiteren Sinne des Wortes als Chromidien (Hertwig. Schaudinn. Goldschmidt, Bluntschli etc.) auch in der Zelle selbst vorkommen und sich demgemäß an Ort und Stelle veländeln können, sodal im Sinne der älteren Zytologie ein direkter Nachweis eines Uebertrittes der ersten Stufen der Körper aus dem Kern ins Protoplasma, für den einige Bilder zu sprechen scheinen, nicht einmal mehr notwendig ist. Sicher sind sie aber nicht blobe ausgestobene Nukleolen (Berlinerblaumethode negativ) noch inoculierte Leukocyten oder Kerntrümmer selbst - all dieses soll in del ausführlichen Arbeit näher bewiesen werden. Nach Mallory färben sie sich gelbrot, wie gewisse Inhaltskörper del Kerne der Uebergangszone. In der Kaninchencornea kolnmen tatsächlich auch Chromidien vor, die besonders in den Basalzellen nachweisbar sind (Giemsafärbung. Saffranin. Viktoriablau etc.) und die zum Teil auch von Gorini beubachtet wurden. Sie beteiligen sich nicht an der eigentlichen Mitose. Die Guarnierikörpel degenerielen oft $\mathrm{zu}$ eigenartigen, mit Ausflockungssediment elfullten blassen Kugeln 
oder zerplatzen raketenartig in der Zelle ( $\mathrm{H}$ ü ckel), die später vakuolig entartet ist. Da beim Kaninchen in der Cornea die Immunität lokal histogen, ja rein zellular ist, so dürfte man in ihnen vielleicht die Träger der immunisierenden Substanzen erblicken.

VII. In der Kaninchencornea durchläuft das Virus nicht nach Art der Protozoen einen Entwicklungszyklus; denn man kann mit allen Stadien von 8 bis 336 Stunden in gleicher Weise ohne eine wesentliche Aenderung der Inkubationszeit erfolgreich impfen.

VIII. Was die Verteilung des vermutlichen Erregers im Epithel anbelangt, so scheint er zunächst nicht allein an das Protoplasma der Zellen gebunden zu sein, sondern kommt bald auch in den Intrazellularen derselben vor. Dafür scheint folgendes Experiment zu sprechen: Läßt man eine 16 Stunden bis mehrere Tage lang infiziert gewesene Cornea etwa 8 bis 10 Stunden ausgeschnitten in einer sterilen Glasdose liegen, so runden sich die einzelnen Zellen nach einiger Zeit ab, indem die mehrfach beobachtete Framboisia externa (Roux) eintritt. Hierauf kann man viele Zellen, ohne sie zu verletzen, einfach in ein Uhrschälchen mit steriler physiologischer Kochsalzlösung abschütteln. Filtriert man nun diese Flüssigkeit durch ein vierfaches Filterpapier, auf dem die Zellen zurückgehalten werden, so kann man trotzdem mit dem Filtrat infizieren. Dagegen scheint das Virus in den Kernen der Zellen später kaum häufig vorzukommen; denn schloB man die Zellen durch partielle Trypsinverdauung auf, filtrierte scharf in der oben angeführten Weise, so konnte man mit dem Rückstand nicht mehr in deutlicher Weise infizieren.

IX. Las Virus kreist nach der Corneainfektion nicht wie beim Kalb im Kaninchenkörper; weder nach einer einfachen noch nach der doppelten Corneainfektion von mehreren Stunden bis 57 Tagen noch nach einer gleichzeitigen ( $7-50$ stündigen bis achttägigen) intraperitonealen lnjektion konnte mit dem Blut, dem PreBsafte aus den Nieren oder der Peritonealflüssigkeit ( $\bar{i}$ Stunden) mit Erfolg eine oculare Infektion vorgenommen werden. Stets wurden mehrere Augen gleichzeitig mit denselben Flüssigkeiten geimpft. Einzelne Augen wurden zur Kontrolle zu Präparaten verarbeitet, in denen keine Guarnierischen Körper nachgewiesen werden konnten. Der Rest konnte später immer wieder mit Erfolg mit der normalen Lymphe infiziert werden (39 Experimente). In diesem Sinne stimmen meine Befunde mit denen von $J$ ürgens (Berliner klinische Wochenschrift No. 11) überein und sprechen gegen die Annahme von Siegel.

Die Preßsäfte wurden in einem eigens konstruierten, nicht dicht schließenden Stempelzylinder unter mäßigem Druck gewonnen. Die Parasiten können bei diesem vorsichtigen Verfahren nicht geschädigt werden; denn in der Cornea können sie einen ziemlich starken Druck vertragen, wie man mit einem der A tw o odschen Fallmaschine ähnlichen Apparat nach der Formel: $\frac{9 \cdot 81 \mathrm{~h} \mathrm{~m}^{2}}{\mathrm{~m}+2 \mathrm{M}}$ Erg. numerisch berechnen kann. Nach einem Druck von 3000 Erg. erhielt ich noch an zwei Stellen der Cornea eine leichte Infektion. Neben einzelnen seltenen, großen, pilzartigen Formen (intraperitoneale Infektion) konnte ich in einzelnen, wenn auch nicht allen Fällen den Siegelschen Körpern ähnliche Gebilde, die ich für saprophytische Nebenorganismen halten möchte, konstatieren - ein Befund, der ausblieb, sobald ich mit einer älteren, drei Tage bei Bruttemperatur gehaltenen, später noch nach Green mit Chloroformdämpfen behandelten Lymphe arbeitete, die, wie ich mich überzeugen konnte, so aber nur keimarm gemacht wurde. Die Züchtung dieser Körper gelang mir weder nach dem Verfahren von Novy (Trypanosomenzüchtung) noch auf Glykogenagar bei Bruttemperatur. An Nierenschnitten bemerkt man manchmal kleinste Gewebeläsionen (ein bis zwei Zellen), durch die ein Austritt von Keimen im Sinne von Biedl und Kraus, Futterer, Pawlowsky u. a. erklärlich wäre. In der Milz gehen oft viele pseudoeosinophile Leukocyten zugrunde, deren Kerne und Nukleolen zu eigentümlichen Körpern zusammenschmelzen.

$X$. Mit dieser Erscheinung hängt wohl aufs innigste die eigenartige lokale Imununität des Kaninchens zusammen.
Nach der Impfung und dem Ausheilen des einen Auges konnte ich nach mehreren Stunden bis nach zwei Monaten immer das andere Auge infizieren. Die einmal infiziert gewesene Stelle konnte ich dagegen nicht mehr impfen; Impfungen in der nächsten Nähe (nach drei Wochen bis zwei Monaten) riefen nur eine leichte Facettierung der geschädigten Stelle hervor, die alsbald verschwand. Die lokale Immunität wurde auch erzielt, wann man die Leukocyten durch wiederholte seitliche Tuscheinjektionen gleichsam auf andere Stellen lockte und überdies durch (14 tägige) Beeinflussung der infizierten Stelle mit einer Opiumlösung nach dem Vorgang von Cantacuzène die Diapedese der Leukocyten verzögerte. Nach noch nicht abgeschlossenen Versuchen kann man durch subcutane Injektion auch ein präzipitierendes antivirulizides Serum gewinnen (Freyer).

Die Aktivität einer infizierten Cornea wird durch ent. sprechende Mengen von Zellmaterial, das einer immunisierten Cornea entstammt, nur dann unwirksam gemacht, wenu man die fraglichen Zellen, in denen die Immunkürper sitzen. mechanisch oder durch Trypsin aufschliebt und dann die beiden Zellderivate mischt, um in der Folge mit diesem Material Kontrollimpfungen vorzunehmen.

XI. Da nach diesen Untersuchungen die Vaccineerkrankung beim Kaninchen ganz lokalisiert werden kann, so eignet siclı aus eben diesem Grunde das genannte Tier in hohem Grade für das Studium des fraglichen Erregers; ferner kann man nach dem Vorgang von Wasielewski generationsweise (46 Generationen) die wirksame Vaccine im Corneaepithel des Kaninchens fortzüchten - eine Beobachtung, die ich völlig bestätigen kann - und ist so berechtigt, geradezu von einer lokatlisierten, organischen Kultur des Erregers zu sprechen.

Die ausführlichere Arbeit wird in den „Arbeiten aus dell Kaiserlichen Gesundheitsamte" später erscheinen. 